LA W RENCE LIVERMORE NATIONAL LABORATORY

Ground Model Options in the NEC-4.2 Antenna Code

G. J. Burke

July 6,2011 
This document was prepared as an account of work sponsored by an agency of the United States government. Neither the United States government nor Lawrence Livermore National Security, LLC, nor any of their employees makes any warranty, expressed or implied, or assumes any legal liability or responsibility for the accuracy, completeness, or usefulness of any information, apparatus, product, or process disclosed, or represents that its use would not infringe privately owned rights. Reference herein to any specific commercial product, process, or service by trade name, trademark, manufacturer, or otherwise does not necessarily constitute or imply its endorsement, recommendation, or favoring by the United States government or Lawrence Livermore National Security, LLC. The views and opinions of authors expressed herein do not necessarily state or reflect those of the United States government or Lawrence Livermore National Security, LLC, and shall not be used for advertising or product endorsement purposes.

This work performed under the auspices of the U.S. Department of Energy by Lawrence Livermore National Laboratory under Contract DE-AC52-07NA27344. 


\section{Ground Model Options in the NEC-4.2 Antenna Code}

\section{Introduction}

The antenna modeling code NEC-4 includes a capability for modeling antennas above or in a homoeneous ground using interpolation in tables of Sommerfeld integrals and asymptotic approximations for large distances. This model has been validated in a number of ways and yields accurate results for common antennas such as monopoles on ground stakes and ground screens and horizontal wires very close to the earth. The original NEC-4 Sommerfeld interpolation was set up for typical ground parameters and has difficulties covering the range of conductivity up to seawater at lower HF. Errors are sometimes seen in plots as discontinuities or spikes, especially in the $H$ field which is obtained from a numerical evaluation of the curl of $E$. A new ground option has now been added in the version NEC-4.2 with a more accurate interpolation method and asymptotic approximations and direct evaluation of $H$. Filling the interpolation tables takes somewhat longer than in the original method, but time to fill the matrix is about the same. Since desktop computers are now more than an order of magnitude faster than the large computers used when NEC-4 was first released in 1992, it is reasonable to trade some speed for accuracy. The original Sommerfeld/interpolation method, which is still available, uses the input option GN2 while the new method is obtained with GN3. Some initial results of the new method are compared with the old solution here.

\section{Discussion}

A key problem in the interpolation of fields due to ground is to interpolate accurately over the wiggles in the real and imaginary parts of the fields while keeping the number of sample points low to minimize computation time. For some ranges of source and evaluation points the fields can be approximated by a single ray, and much of the phase variation can be removed by diving out a phase factor for that ray, leaving a relatively smooth surface for interpolation. However, along the interface an asymptotic form may require two rays that interfere, so dividing out the phase variation becomes much more difficult. Interference of the rays can result in zeros, ruling out division. The original NEC-4 solution dealt with this problem by using expressions modeling the field variation of the two rays and fitting the sum to computed field values using a least-squares algorithm. The models used in fitting include the asymptotic phase factors and algebraic factors determined for each field component by extensive numerical testing. For small distances, before the phase variation becomes significant, simple interpolation is used after extracting quasistatic field terms, and at large distances greater than about two wavelengths asymptotic approximations are used. These approximations were done only for the $E$ field, which is the core of the NEC- 4 wire solution. The $H$ field is obtained by a numerical difference evaluation of the curl of $E$, which magnifies any errors in the $E$ fields and results in the most glaring "glitches".

The new GN3 option uses only cubic interpolation with more interpolation regions and evaluates both $E$ and $H$ directly. The density of sample points is determined adaptively for the particular ground parameters and is higher than with the GN2 method. In regions where significant interference patterns occur the two branch cut integrals are evaluated and interpolated separately. The branch cut integrals have simple phase variations when they represent distinct rays, so the phase can be divided out. In regions of space where interference is possible the interpolation accuracy is tested for total and branch-cut interpolation and 
index tables are set up to determine which will be used.

The GN3 option also has more accurate asymptotic approximations for large distances. GN2 uses a second-order asymptotic formula for the ray above ground but only first order for the ray below ground. The first order asymptotic goes to zero when the ray becomes grazing to the interface, so the interference of the rays at grazing incidence is lost. GN3 uses a higher order approximation along the interface that preserves the interference. A new higher order asymptotic approximation is also used for small $\rho$ and large $z$ where the usual asymptotic approximations fail because the large argument forms of the Bessel functions cannot be used. The GN2 solution used a kludge of interpolating higher order terms from a second order approximation valid only for $\rho=0$ and the higher order terms for larger $\rho$.

Since GN3 uses more sample points in the interpolation and has the complexity of separating branch cuts it takes longer to fill the tables. The time depends on the number of tables needed for the spatial extent of the model and field evaluation points, but can be several tens of seconds. For GN2 the time to fill the tables could be less than a second. GN3, like GN2 can save the tables to a file, so if the same problem is run again the tables are obtained very quickly.

\section{Results Comparing the Ground Models}

An initial test model was a whip antenna with $5 \mathrm{~m}$ height and 10 segments on a ground stake of $0.2 \mathrm{~m}$ depth and 6 segments in the sea. The wire radius was $0.005 \mathrm{~m}$, small enough to avoid any thin-wire problems on the ground stake. Seawater parameters were taken as $\epsilon_{r}=$ 81 and $\sigma=4 \mathrm{~S} / \mathrm{m}$. This results in a complex relative permittivity of $81-j 14380$ at $5 \mathrm{MHz}$. The computed input impedance was 58.76 - j765.42 ohms with GN2 and $58.88-j 765.56$ ohms with GN3.

Results for the $E$ and $H$ fields for increasing height $z$ at a distance from the monopole of $x=1 \mathrm{~m}$ are shown in figures 1 and 2. Fields from GN2, GN3 and also perfectly conducting ground are compared. A region of increased error in the GN2 result is seen in $E_{z}$ for $z$ less than about $0.5 \mathrm{~m}$. This results in a large error in $H_{y}$, which is obtained from a finitedifference evaluation of $\nabla \times E$. Increased errors are also seen in $H_{y}$ for $z$ greater than 4.5 $\mathrm{m}$. Although the $E$ fields appear to be in good agreement in this range the differencing and possible cancelation of $\partial E_{x} / \partial z$ and $\partial E_{z} / \partial x$ terms in the curl can magnify errors. Plots for this same model with $x$ increasing with $z=0.1 \mathrm{~m}$ are shown in figures 3 and 4 . Increased error with GN2 is seen in $E_{z}$ for $x$ from about .5 to $2 \mathrm{~m}$. Errors in $H_{y}$ are substantial through this range. In all cases the GN3 results are in close agreement with the PEC ground result for this seawater ground.

Similar plots are shown in Figures 5 through 8 for the same monopole model with low conductivity water with $\epsilon_{r}=81$ and $\sigma=0.01 \mathrm{~S} / \mathrm{m}$. The agreement between GN2 and GN3 results for $E$ is much better with this lower conductivity, although $H_{y}$ still shows some errors from differencing. Larger differences are now seen between the water and PEC ground results as expected.

Fields for a short vertical dipole over zero conductivity water are shown in Figures 9 through 11 scaled to $1 \mathrm{~m}$ wavelength $(299.8 \mathrm{MHz})$. The dipole extends from $0.002 \lambda$ to $0.012 \lambda$ above the water. The $E_{x}$ field shows the interference along the interface from fields in both media which disappears when GN2 switches to asymptotic for $x>2 \lambda$. GN3 switches 

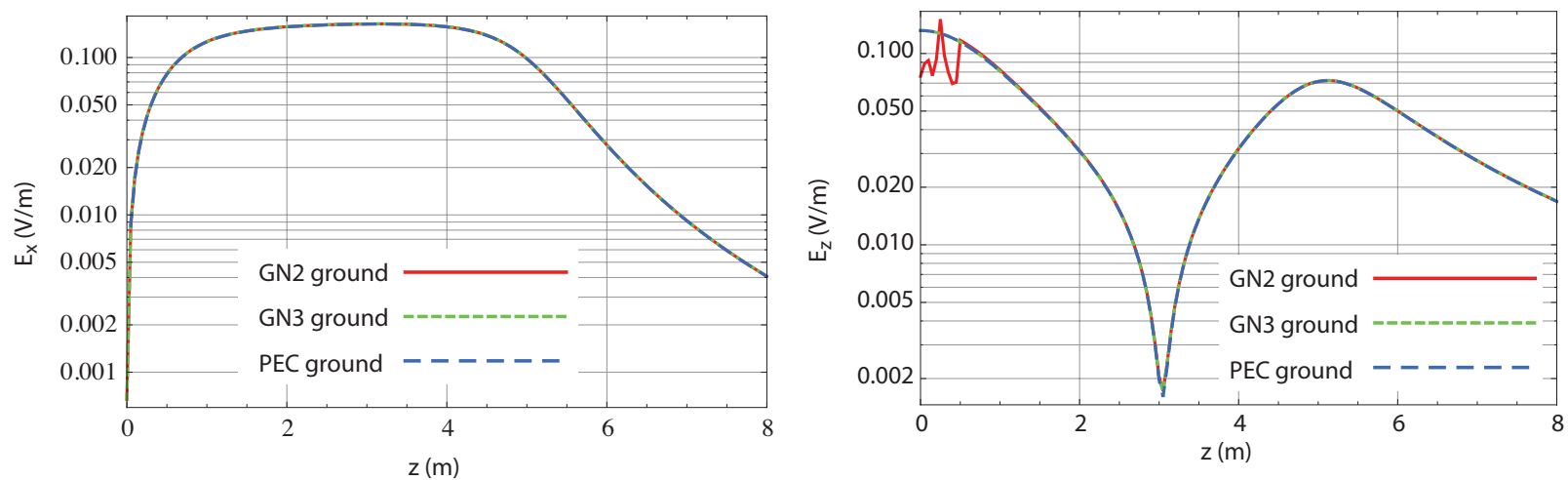

Fig. 1 Near $E$ fields versus z for $\mathrm{x}=1 \mathrm{~m}$ for a $5 \mathrm{~m}$ monopole on a seawater ground at $5 \mathrm{MHz}$.

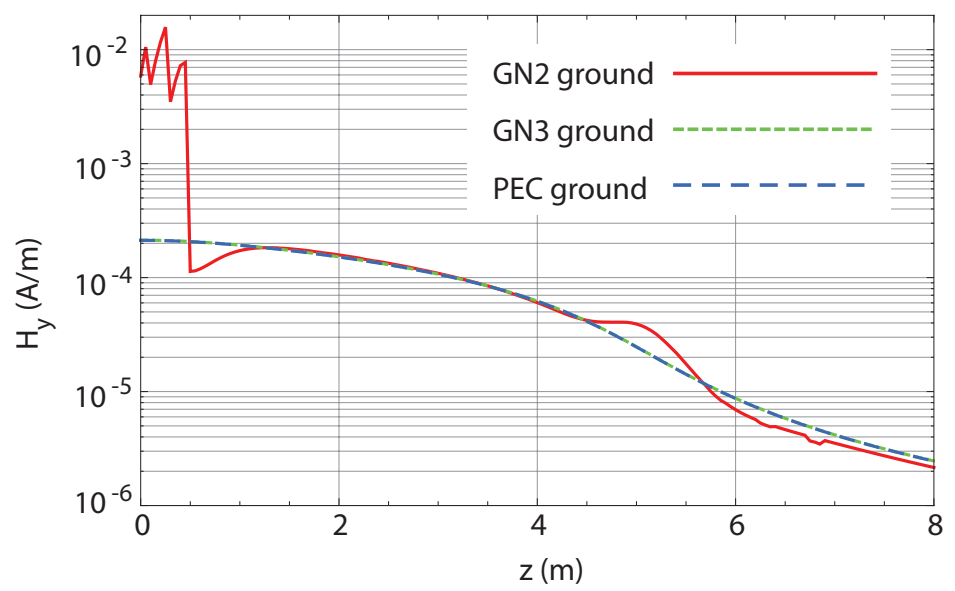

Fig. 2 Near $H_{y}$ field versus z for $\mathrm{x}=1 \mathrm{~m}$ for a $5 \mathrm{~m}$ monopole on a seawater ground at $5 \mathrm{MHz}$.

to asymptotic for $x>3 \lambda$, but the interference continues. The GN2 result for $H_{y}$ shows an isolated glitch around $x=0.6 \lambda$, but is in good agreement above this point.

\section{Conclusion}

The GN3 option for ground in NEC-4.2 provides a more accurate evaluation of the fields than GN2 at the expense of somewhat longer time to fill the interpolation tables. The GN2 model should give accurate results for most typical antennas and the normal range of ground parameters. GN3 may be needed for high conductivity ground and for accurate evaluation of the near $H$ field. The GN3 model differs from GN2 in the evaluation of the Sommerfeld integrals, in the interpolation and in the integration over the segment currents, so it provides a fairly independent check on the GN2 result.

The NEC-4.2 code also includes a new model for current sources (EX6) that can be used like the NEC-4 "applied field" voltage sources. It also allocates and reallocates all arrays as needed, so there should be no fixed limits on model size or number of input commands. 

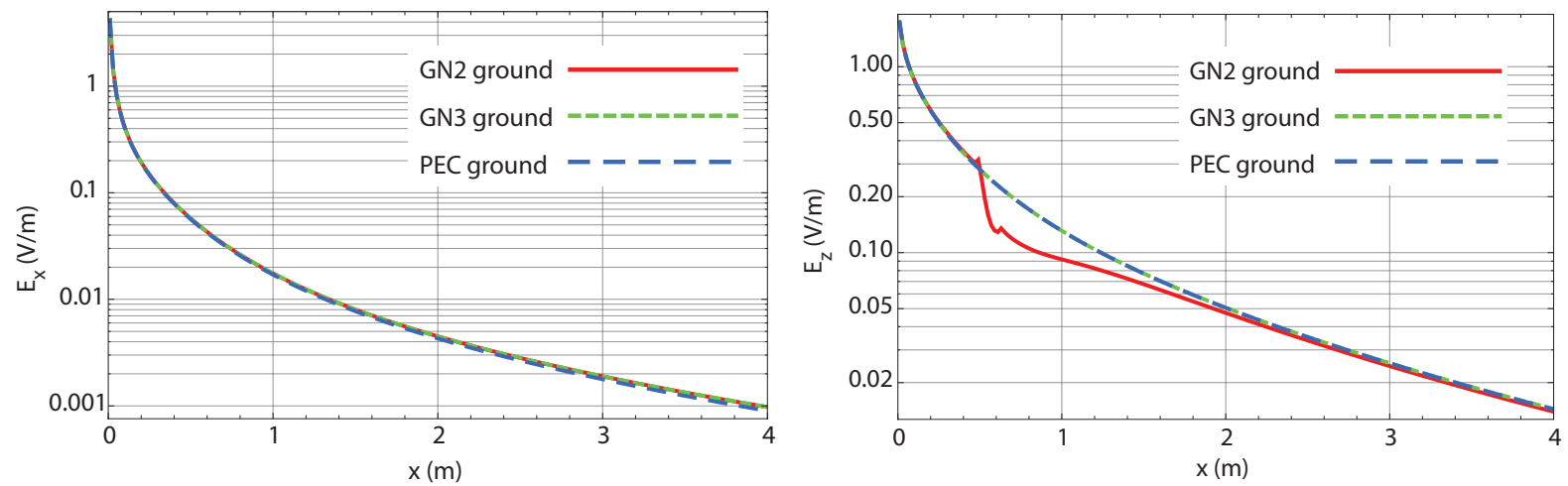

Fig. 3 Near $E$ fields versus $\mathrm{x}$ for $\mathrm{z}=0.1 \mathrm{~m}$ for a $5 \mathrm{~m}$ monopole on a seawater ground at $5 \mathrm{MHz}$.

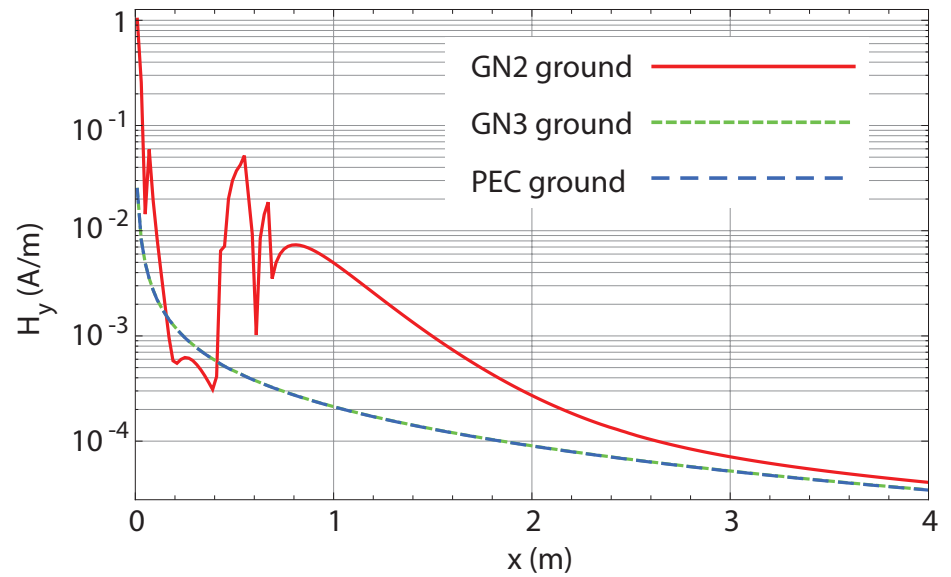

Fig. 4 Near $H_{y}$ field versus $\mathrm{x}$ for $\mathrm{z}=0.1 \mathrm{~m}$ for a $5 \mathrm{~m}$ monopole on a seawater ground at $5 \mathrm{MHz}$.
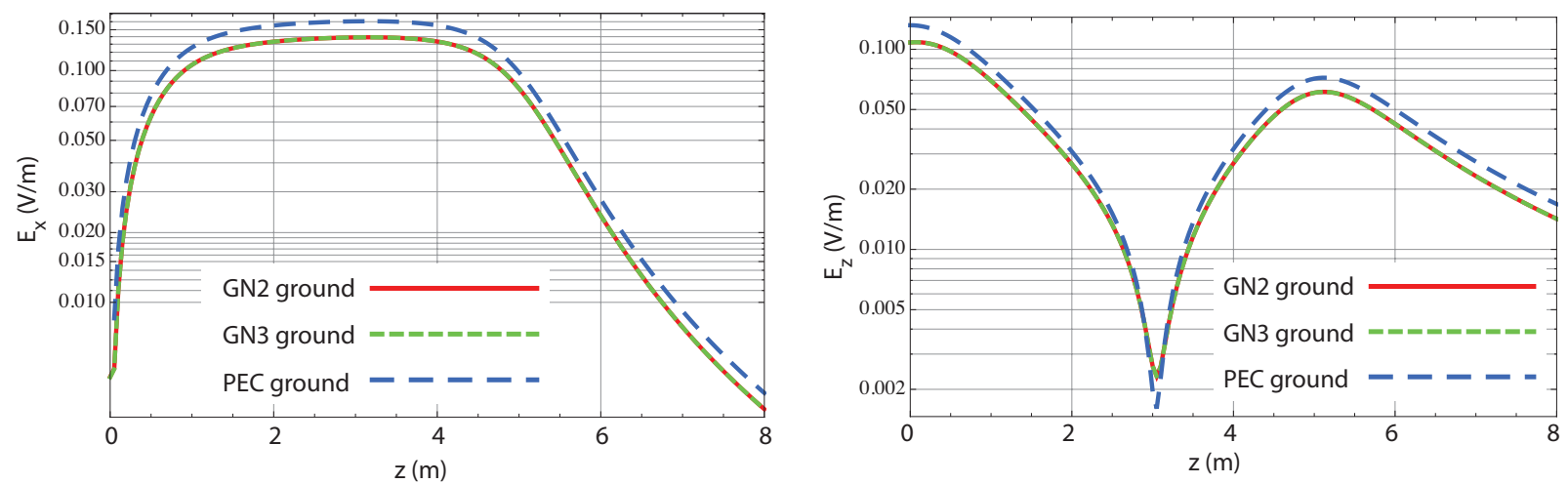

Fig. 5 Near $E$ fields versus z for $\mathrm{x}=1 \mathrm{~m}$ for a $5 \mathrm{~m}$ monopole on low conductivity water at $5 \mathrm{MHz}$. 


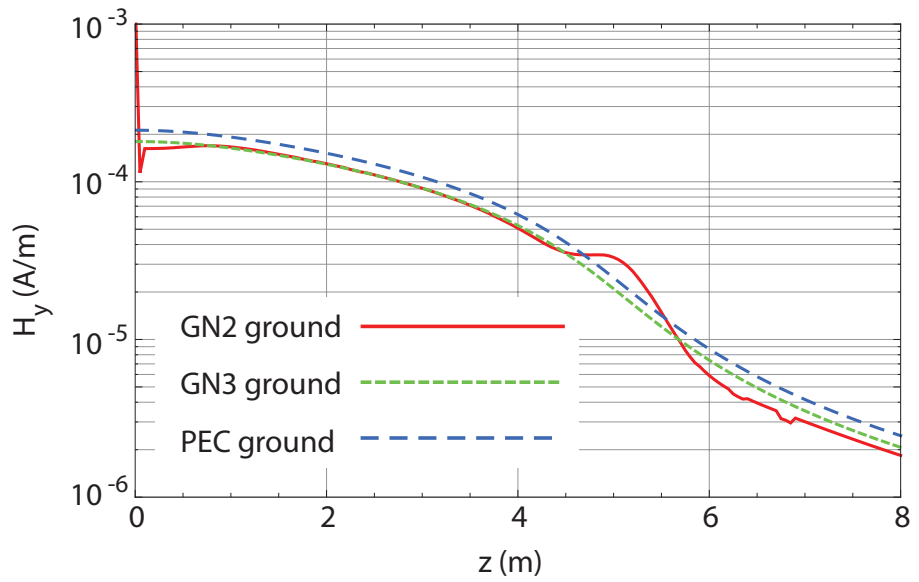

Fig. 6 Near $H_{y}$ fields versus z for $\mathrm{x}=1 \mathrm{~m}$ for a $5 \mathrm{~m}$ monopole on low conductivity water at $5 \mathrm{MHz}$.
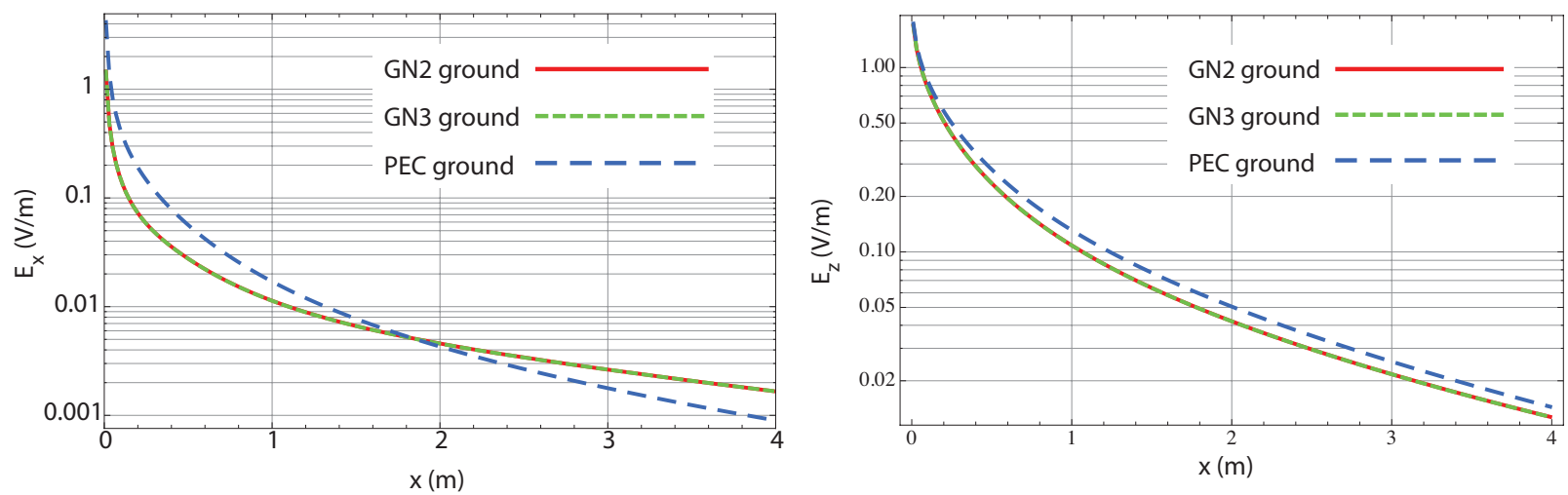

Fig. 7 Near $E$ fields versus $\mathrm{x}$ for $\mathrm{z}=0.1 \mathrm{~m}$ for a $5 \mathrm{~m}$ monopole on low conductivity water at $5 \mathrm{MHz}$.

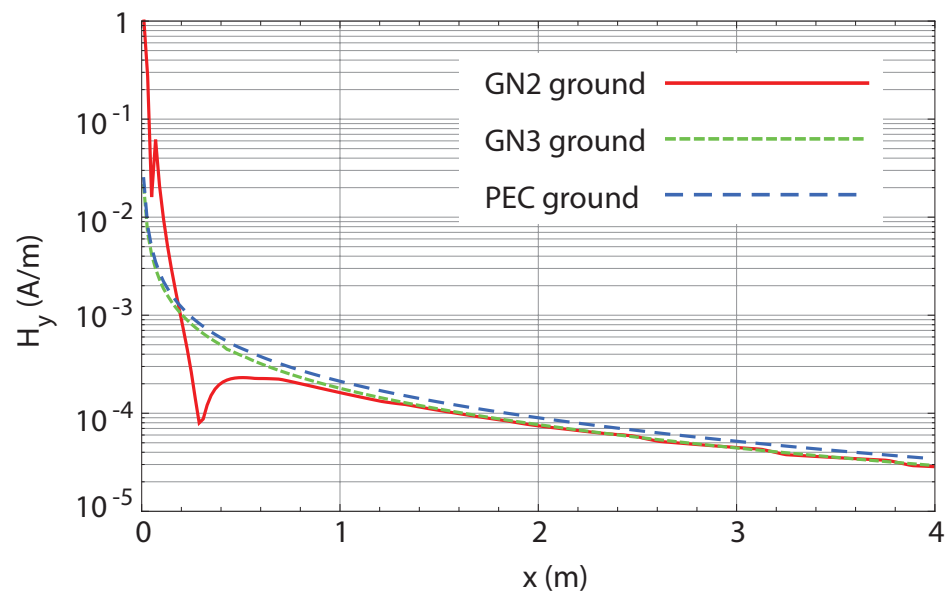

Fig. 8 Near $H_{y}$ field versus $\mathrm{x}$ for $\mathrm{z}=0.1 \mathrm{~m}$ for a $5 \mathrm{~m}$ monopole on low conductivity water at $5 \mathrm{MHz}$. 

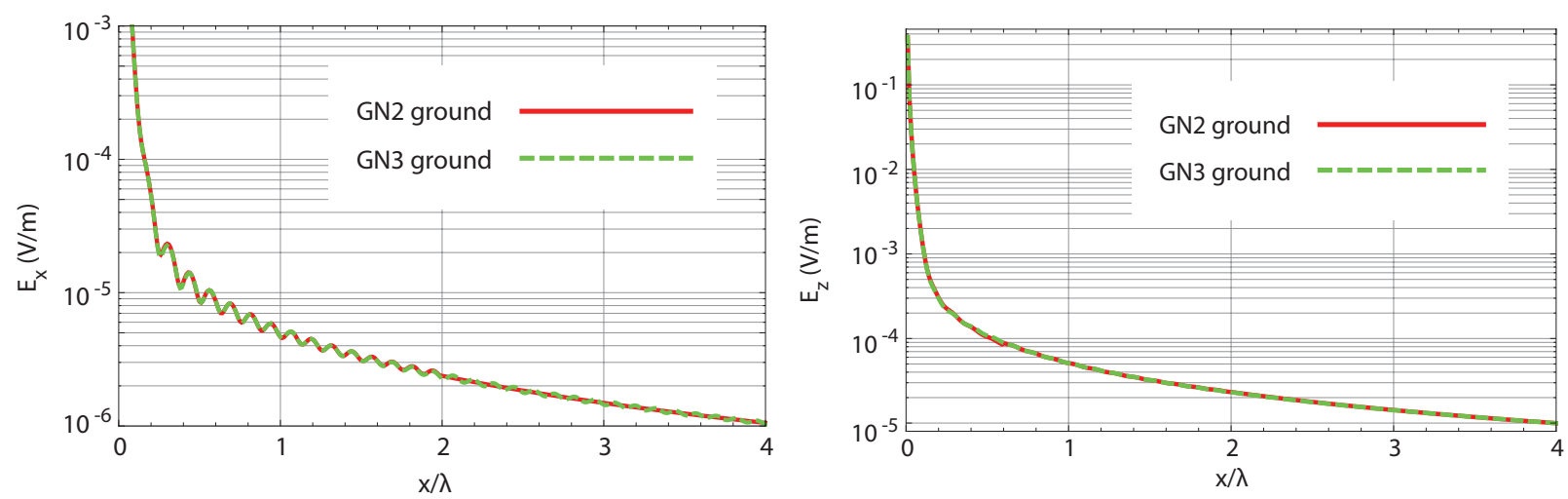

Fig. 9 Near $E$ fields versus $x$ for $z=0.01 \lambda$ for a short vertical dipole over zero conductivity water.

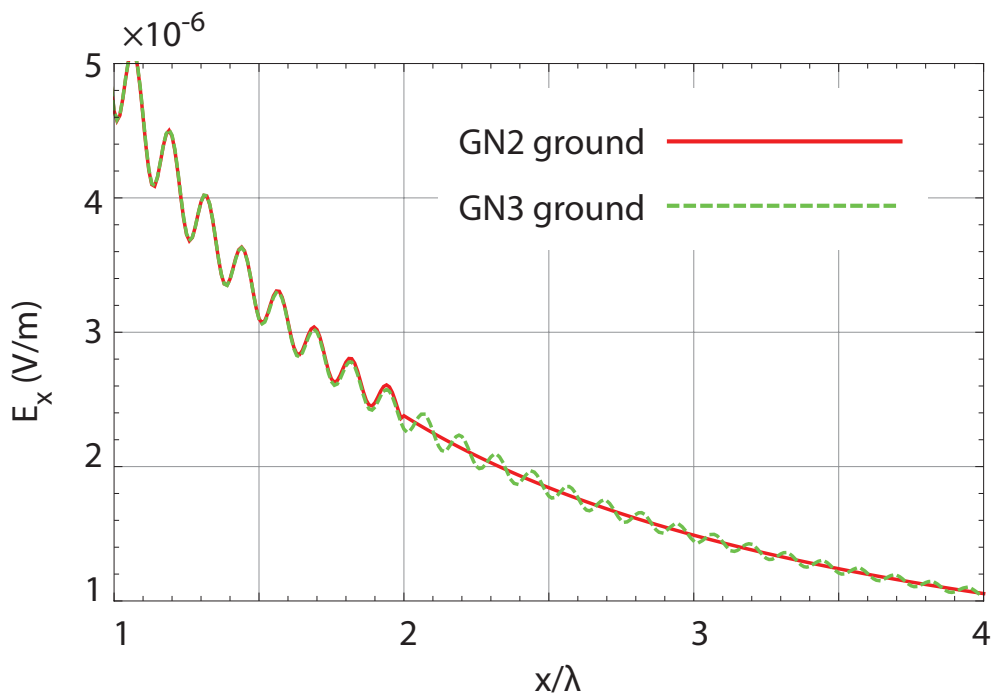

Fig. 10 Near $E_{x}$ versus $x$ for $z=0.01 \lambda$ for a short vertical dipole over zero conductivity water (expanded scale). The interference disappears when GN2 switches to asymptotic.

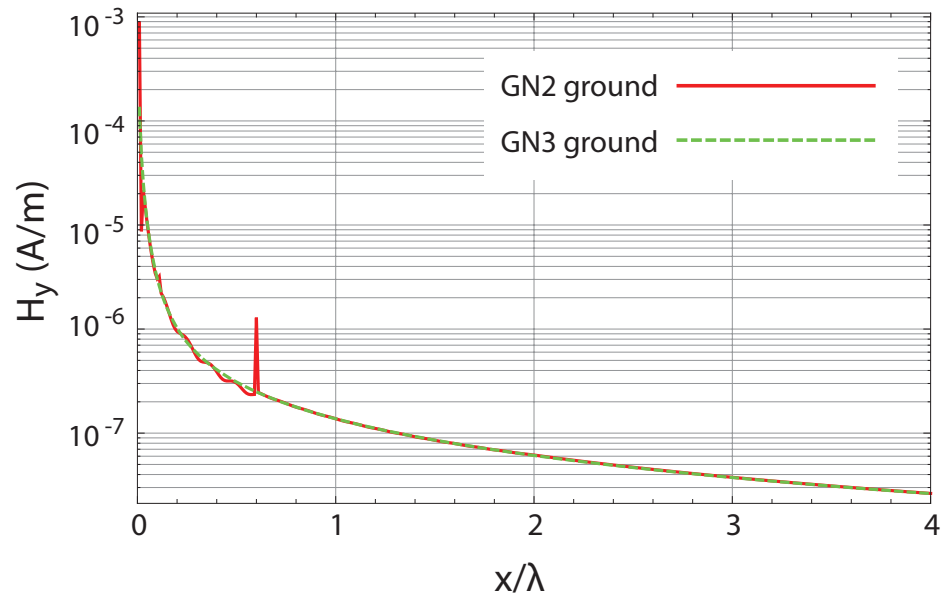

Fig. 11 Near $H_{y}$ field versus $x$ for $z=0.01 \lambda$ for a short vertical dipole over zero conductivity water. 